Research Paper

\title{
Prognostic significance of serum soluble DR5 levels in small-cell lung cancer
}

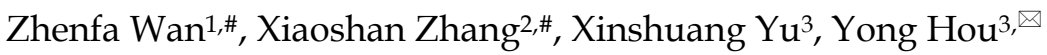 \\ 1. Department of Medical Imaging, The Fourth Hospital of Jinan City, Shandong, 250014, China \\ 2. Department of Medical Imaging, Weihaiwei People's Hospital, Weihai, Shandong, 264200, China \\ 3. Department of Radiation Oncology, Shandong Provincial Qianfoshan Hospital, Jinan, Shandong, 250014, China \\ \#These two authors contributed equally to this work. \\ $\triangle$ Corresponding author: Dr. Yong Hou, Department of Radiation Oncology, Shandong Provincial Qianfoshan Hospital, 16766 Jingshi Road, Lixia District, \\ Jinan, Shandong 250014, China. Tel.: +86-15505412654; E-mail: 15562689543@163.com \\ (C) Ivyspring International Publisher. This is an open access article distributed under the terms of the Creative Commons Attribution (CC BY-NC) license \\ (https://creativecommons.org/licenses/by-nc/4.0/). See http://ivyspring.com/terms for full terms and conditions.
}

Received: 2018.07.27; Accepted: 2018.12.20; Published: 2019.01.29

\begin{abstract}
The death receptor 5 (DR5) is a member of the tumor necrosis factor receptor superfamily that can transduce the apoptosis signal in cells. This study assessed serum levels of soluble death receptor 5 (sDR5) in small-cell lung cancer (SCLC) patients compared with those in healthy controls. Clinicopathological features of patients, treatment responses, and overall survival of patients were also recorded and analyzed. The sDR5 levels were analyzed using ELISA in 50 healthy controls and 82 SCLC patients before and after first-line chemotherapy. The statistical data showed that pre-treatment levels of serum sDR5 in SCLC patients were higher than those of healthy controls $(P<0.001)$. Pre-treatment levels of serum sDR5 were significantly associated with smoking history of patients, Veterans Administration Lung Study Group (VALSG) stage, tumor size, and lymph node $(N)$ metastasis $(P=0.028,0.001,0.028$, and 0.01 , respectively). After treatment with the first-line chemotherapy, the post-treatment levels of serum sDR5 were obviously decreased $(P<0.001)$, and correlated with treatment responses $(P<0.001)$, although there was no significant difference in their pretreatment sDR5 levels $(P=0.62)$. Cox proportional hazard analysis demonstrated that the post-treatment levels of serum sDR5, VALSG stage, and PS status were all independent predictors for overall survival of patients. The results from the current study indicate that serum level of sDR5 could be further confirmed as a biomarker to predict treatment responses and survival of SCLC patients.
\end{abstract}

Key words: Small-cell lung cancer, soluble death receptor 5, biomarker, treatment responses, overall survival

\section{Introduction}

Lung cancer remains one of the most commonly diagnosed human cancers in the world. Lung cancer can be histologically classified into small-cell lung cancer (SCLC) and non-small cell lung cancer (NSCLC) [1, 2]. SCLC only represents a small portion (approximately 13\%) of all lung cancer cases, but it is clinically aggressive and associated with early extrathoracic metastases [3]. SCLC cells contain dense neurosecretory granules along with neuroendocrine hormones that induce endocrine/paraneoplastic syndrome [3]. Although SCLC responds well to chemotherapy [4], it is frequently diagnosed at an advanced stage thus leading to a poor prognosis [5]. The median survival without treatment is only 2 to 4 months and overall 5-year survival rate is approximately $3-8 \%[6,7]$. To date, platinum- and etoposide-based chemotherapy is the first-line treatment [8, 9], but tumor recurrence and metastases lead to a dismal prognosis [10]. Therefore, a search for novel therapeutic strategies or biomarkers for early detection and prediction of prognosis and treatment responses could lead to better control of this deadly disease.

Tissue homeostasis is the balance of cell proliferation and death (apoptosis) [11], while tumor development and progression are characterized by an increase in cancer cell growth and evasion of tumor cell apoptosis [12, 13]. Tumor necrosis factor 
(TNF)-related apoptosis-inducing ligand (TRAIL) is a member of the TNF superfamily that can induce tumor cells to undergo apoptosis [14-16]. Activated TRAIL binds to the death receptor and subsequently induces caspase activation and cell apoptosis [14-16]. To date, five death or decoy receptors have been discovered that bind to TRAIL: death receptor 4 (DR4 or TRAIL-R1), DR5 (or named as TRAIL-R2), DcR1 (TRID/TRAIL-R3), DcR2 (TRAIL-R4), and the soluble receptor osteoprotegerin [17]. DR5 can transduce the apoptosis signal in cells [18, 19], but DR5 overexpression has been indicated in several types of human cancers, including colon, lung, cervical cancers and head and neck squamous cell carcinoma [16, 20-24]. Another previous study showed that the endoplasmic reticulum (ER) stress induced DR5 transcription and apoptosis in colon cancer HCT116 cells [25], although soluble DR5 (sDR5) levels were upregulated and significantly associated with poor prognosis of patients with NSCLC [26]. Thus, in this study, we first assessed serum sDR5levels in SCLC versus healthy controls, investigated the clinicopathological features of SCLC patients, and predicted treatment responses and overall survival of patients. We sought to provide insightful information regarding sDR5 as a biomarker to predict treatment responses and overall survival of SCLC patients.

\section{Patients and methods}

\section{Patients}

In this retrospective case-control study, we obtained serum samples from 82 SCLC patients (47 males and 35 females) who underwent treatment in Shandong Provincial Qianfoshan Hospital (Jinan, China) between January 2010 and April 2013. All patients were histologically or cytologically confirmed as SCLC cases; however, any patients who received surgery were excluded from this study, while patients receiving any previously systemic therapy within 6 months were also excluded. The SCLC patients were staged according to the Veterans Administration Lung Cancer Study Group (VALSG) staging system [27] and all patients were required to have a dimensionally measurable disease through chest radiograph or computed tomographic (CT) scanning. The patients were randomized to receive standard (up to 6-cycle) treatment with etoposide and cisplatin (EP) or etoposide and carboplatin (CE), with or without radiotherapy. The therapeutic dose was adjusted according to each patient's physical condition. Patients were followed up regularly with physical examinations, blood chemistry analysis, ultrasound examination of the abdomen, X-ray or CT scanning of the chest, brain CT or MRI, and scintigraphy of the skeleton.
In addition, we also collected blood samples from 50 healthy controls who visited our hospital for annual health check-ups and who were matched to the SCLC cases by age and gender. The study was approved by the Ethics Committee of Shandong Provincial Qianfoshan Hospital and written informed consent was obtained from each patient.

\section{Evaluation of therapy responses}

All patients received up to 6-cycle randomized treatment with EP or CE with or without radiotherapy and were followed up regularly. The tumor response was measured using the Response Evaluation Criteria In Solid Tumors criteria [28] 4 to 6 weeks after completion of all therapy, which includes complete response (CR, no residual tumor lesions), partial response (PR, more than one-third smaller in tumor lesion), stable disease (SD, no change in tumor lesion) and progressive disease $(\mathrm{PD}$, increase in size of tumor lesion).

These patients were followed up regularly and the last follow-up was conducted on July 1, 2018. We then calculated the overall survival (OS) of each patient, which was defined as duration (months) from disease diagnosis to death or to the patient's last visit. The initial recurrence was categorized as loco-regional recurrence or distant metastasis, depending on location of the recurred tumor lesion.

\section{Enzyme linked immunosorbent assay (ELISA)}

Serum level of sDR5 was assessed by using a solid phase sandwich ELISA kit (cat. \#IB-17792; Jianglai Inc., Shanghai, China) according to the manufacturer's protocol. Our preliminary experiments assessed the detection range of 2-70 $\mathrm{pg} / \mathrm{ml}$ of sDR5 and the A450 value was used to draw the standard curve to normalize the level of sDR5. The ELISA was in duplicate and repeated at least once with similar results.

\section{Statistical analysis}

Serum sDR5 level was summarized as mean \pm standard deviation. Difference between two groups was analyzed by using Student's $t$ test. The Kaplan-Meier curve was used to plot overall survival and the log-rank test was performed to generated $p$ values stratified by sDR5 levels. The Cox's regression model was used to analyze independent predictors for overall survival of these patients against different clinicopathological features and serum level of sDR5. The receiver operating characteristics (ROC) curve was used to analyze the cut-off values of serum levels of sDR5 in patients with SCLC and healthy controls, while the area under the curve (AUC) was also evaluated. A $p$ value $<0.05$ was considered statistically significant. All statistical analyses were performed by 
using SPSS v13.0 software (SPSS Inc., Chicago, IL, USA).

\section{Results}

\section{Patients' characteristics}

Among these 82 SCLC patients, there were 47 males and 35 females with a median age of 55 years old. Forty-three patients were ever tobacco smokers and 39 never smokers, while 48 patients were at LS-SCLC and 34 at ED-SCLC stages, and approximately half of these patients $(n=40)$ had lymph node metastasis of SCLC. Most patients $(n=62)$ received EP chemotherapy and radiotherapy $(n=71)$. After therapy, 59 patients had CR or PR, whereas 23 patients had SD or even PD (Table 1). The median follow-up duration of these patients was 12 months (range between 3 and 33 months), while the median OS was 10 months, with 13 months for LS-SCLC patients and 8.5 months for ED-SCLC patients.

Table 1. Basic characteristics of the study patients

\begin{tabular}{|c|c|c|c|}
\hline & $\mathrm{n}(\%)$ & sDR5 level (pg/ml) & $p$-value \\
\hline Age (years) & & & 0.44 \\
\hline Median (range) & $55(37-76)$ & & \\
\hline$<55$ & $38(46.3)$ & $18.56 \pm 0.74$ & \\
\hline$\geq 55$ & $44(53.7)$ & $19.29 \pm 0.61$ & \\
\hline Gender & & & 0.20 \\
\hline Male & $47(57.3)$ & $19.47 \pm 0.71$ & \\
\hline Female & $35(42.7)$ & $18.25 \pm 0.56$ & \\
\hline PS & & & 0.073 \\
\hline $0-1$ & $63(76.8)$ & $19.42 \pm 0.53$ & \\
\hline $2-3$ & $19(23.2)$ & $17.40 \pm 0.99$ & \\
\hline Smoking history & & & 0.028 \\
\hline Ever smoker & $43(52.4)$, & $19.94 \pm 0.65$ & \\
\hline Never smoker & $39(47.6)$ & $17.86 \pm 0.66$ & \\
\hline Stage (VALSG) & & & 0.001 \\
\hline LS-SCLC & $48(59.5)$ & $17.69 \pm 0.47$ & \\
\hline ED-SCLC & $34(41.5)$ & $20.73 \pm 0.85$ & \\
\hline Tumor size (cm) & & & 0.028 \\
\hline$\leq 3$ & $42.742 .7)$ & $17.75 \pm 0.71$ & \\
\hline$>3$ & $47(57.3)$ & $19.84 \pm 0.61$ & \\
\hline $\mathrm{N}$ status & & & 0.010 \\
\hline positive & $40(47.8)$ & $20.19 \pm 0.66$ & \\
\hline negative & $42(52.2)$ & $17.77 \pm 0.63$ & \\
\hline \multicolumn{4}{|l|}{ Chemotherapy } \\
\hline $\mathrm{EP}$ & $62(75.6)$ & & \\
\hline $\mathrm{CE}$ & $20(24.4)$ & & \\
\hline \multicolumn{4}{|l|}{ Radiotherapy sequence } \\
\hline Concurrent & $55(42.7)$ & & \\
\hline After-chemotherapy & $36(43.9)$ & & \\
\hline None & $11(13.4)$ & & \\
\hline Responses & & & 0.80 \\
\hline$C R+P R$ & $59(72.0)$ & $18.88 \pm 0.53$ & \\
\hline $\mathrm{SD}+\mathrm{PD}$ & $23(28.0)$ & $19.13 \pm 1.03$ & \\
\hline
\end{tabular}

Serum levels of sDR5 were compared by Student's $t$ test or the analysis of variance. Results are presented as the means \pm standard deviation.sDR5, soluble death receptor 5; VALSG, Veterans Administration Lung Cancer Study Group staging system; LS-SCLC, limited-stage small-cell lung cancer; ED-SCLC, extensive-stage small-cell lung cancer; $\mathrm{EP}$, etoposide+ platinum; CE, etoposide + cisplatin; CR, complete response; $\mathrm{PR}$, partial response; $\mathrm{PD}$, progressive disease; $\mathrm{SD}$, stable disease.

\section{Upregulation of serum soluble DR5 level in SCLC patients}

The pre-treatment levels of serum sDR5 in SCLC group were $18.95 \pm 4.80 \mathrm{pg} / \mathrm{ml}$, compared with 10.89 $\pm 6.72 \mathrm{pg} / \mathrm{ml}$ in the 50 healthy controls, indicating that the pre-treatment levels of serum sDR5 were significantly higher in SCLC patients than that of the healthy controls $(\mathrm{P}<0.001$; Figure 1A). However, the level of serum soluble DR5 was downregulated after treating the patients with chemoradiotherapy (18.95 \pm $4.80 \mathrm{pg} / \mathrm{ml}$ vs. $13.54 \pm 3.75 \mathrm{pg} / \mathrm{ml}, \mathrm{P}<0.001$; Figure 1B).

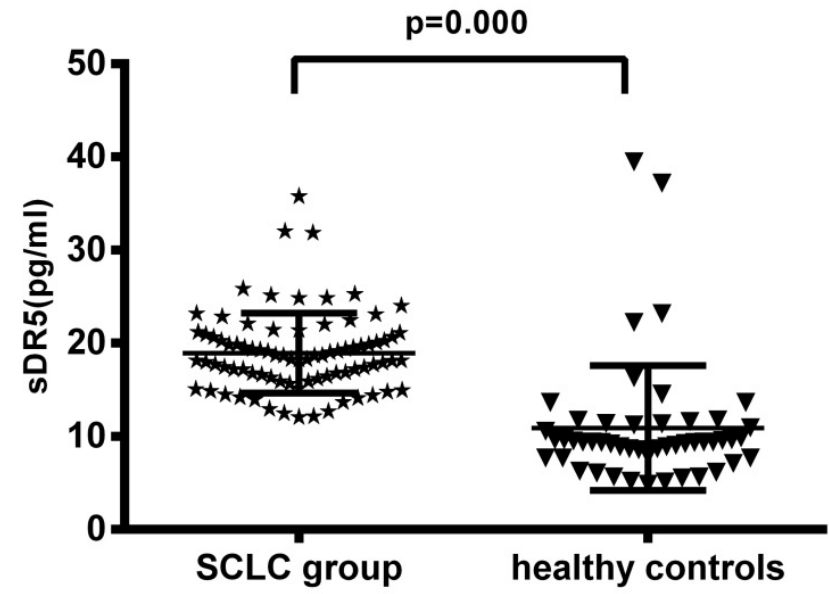

Figure 1. Changes in serum levels of sDR5 in SCLC patients vs. healthy controls or pre-and post-treatment. (A) The blood samples were obtained from 82 SCLC patients and 50 healthy controls and then subjected to analysis of sDR5 levels using the ELISA. $P<0.001$ analyzed by using and Student's $t$ test between SCLC and healthy controls. (B) Association of pre- and post-treatment levels of serum sDR5 in SCLC patients, $P<0.001$ using Student's $t$ test.

\section{Association of pretreatment levels of serum sDR5 with clinicopathological characteristics from SCLC patients}

We then plotted the ROC curve to assess the cut-off value of pretreatment serum sDR5 levels and found $14 \mathrm{pg} / \mathrm{ml}$ as the cut-off value for high vs. low levels of serum sDR5. We then associated sDR5 expression with clinicopathological characteristics from SCLC patients. Our data showed that there was no association of sDR5 expression with age $(\mathrm{P}=0.44$, Figure 2A), gender $(\mathrm{P}=0.207$; Figure $2 \mathrm{~B})$, or performance status (PS; $\mathrm{P}=0.073$ ). However, serum sDR5 level was significantly associated with smoking history, VALSG stage, tumor size, and SCLC lymph node metastasis (Figure 2).

\section{Association of serum sDR5 levels with treatment response}

After treating patients with chemoradiotherapy, the objective response rate (CR + PR) was $72 \%$ (59 out of 82 patients) and the non-response rate (SD + PD) was $28 \%$ ( 23 of 82 patients). We then associated serum 
sDR5 levels with treatment response and found that post-treatment levels of serum sDR5 were significantly associated with treatment responses $(\mathrm{P}<0.0001$; Figure 3A); however, there was no significant association between pre-treatment level of serum sDR5 and treatment responses $(\mathrm{P}=0.62$; Figure 3B).

\section{Univariate and multivariate analyses of OS}

We also performed the univariate and multivariate analyses to predict prognostic predictors for OS of these patients. Our univariate analysis data showed that the performance status, VALSG stages, N stage, post-treatment level of serum sDR5, and tobacco smoking were all prognostic predictors for OS of these patients, while our multivariate analysis demonstrated that the performance status, VALSG stages, post-treatment level of serum sDR5, and tobacco smoking were all prognostic predictors for OS of these patients (Table 2). However, the pre-treatment level of serum sDR5 was not associated with the OS of these patients (Table 2).

We also associated the post-treatment level of serum sDR5 with OS of these SCLC patients using the Kaplan-Meier curves and the log-rank test and found that the reduced post-treatment level of serum sDR5 was associated with better OS of these SCLC patients (Figure 4).
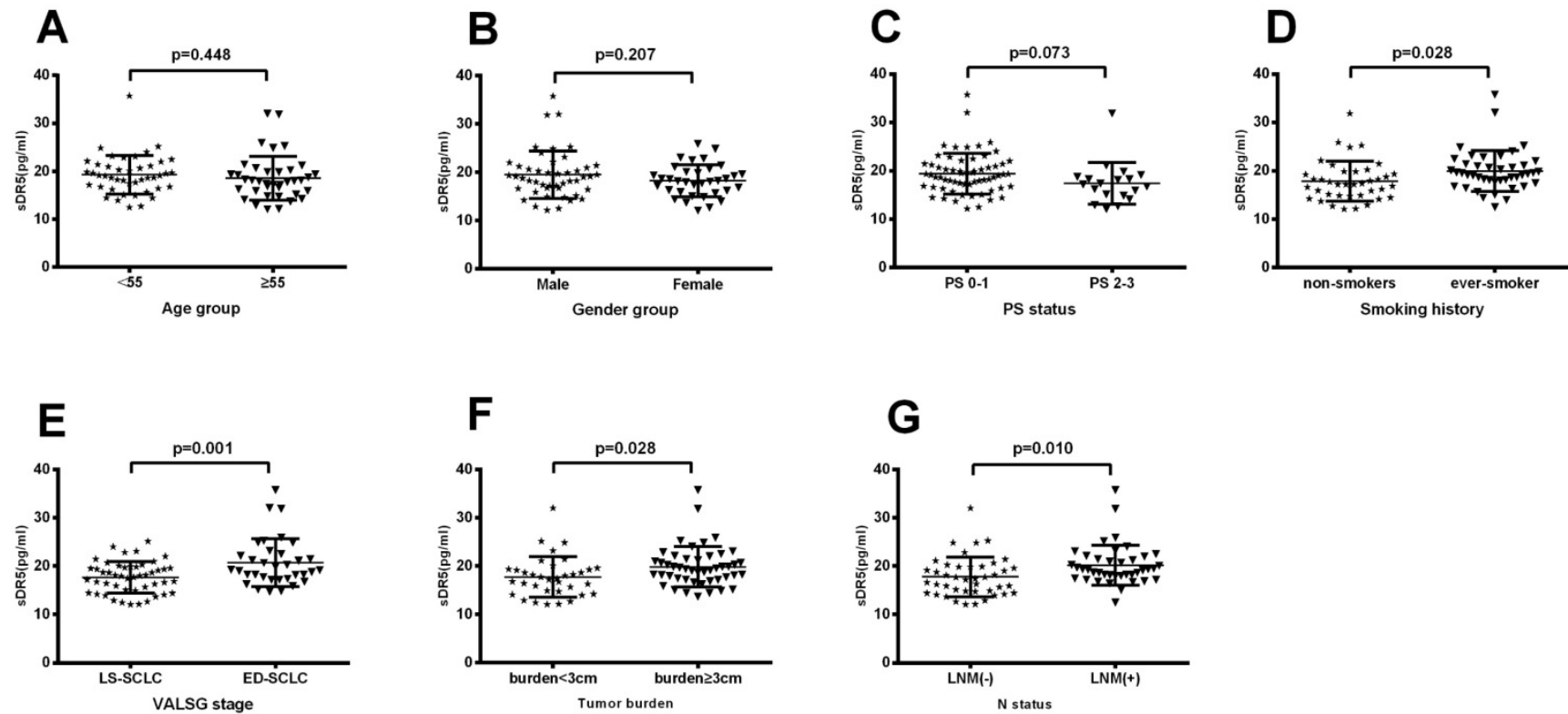

Figure 2. Association of pretreatment serum levels of $s D R 5$ with clinicopathological characteristics from SCLC patients. (A) Pretreatment serum level of $s D R 5$. It compares $<55$ years vs. $\geq 55$ years patient age groups, $P=0.44$. (B) Pretreatment serum level of $s D R 5$. Male patients vs. female patients, $P=0.207$. (C) Pretreatment serum level of sDR5. Patients with PS0-1 vs. PS2-3, $P=0.073$. (D) Pretreatment serum level of sDR5. Patients with never-smokers vs. ever-smokers, $P=0.028$. (E) Pretreatment serum level of sDR5. Patients in LS-SCLC vs. ED-SCLC groups, $P=0.001$. (F) Pretreatment serum level of $s D R 5$. Patients with tumor size of $<3 \mathrm{~cm}$ vs. $\geq 3 \mathrm{~cm}, P=0.010$. (G) Pretreatment serum level of $s D R 5$. Patients with lymph node metastasis vs. none lymph node metastasis, $p=0.01$. PS, performance status; LS-SCLC, limited stage small-cell lung cancer; ED-SCLC, extensive stage small-cell lung cancer; LNM(+), lymph node metastasis positive; LNM(-), lymph node metastasis negative; VALSG, Veterans Administration Lung Study Group.
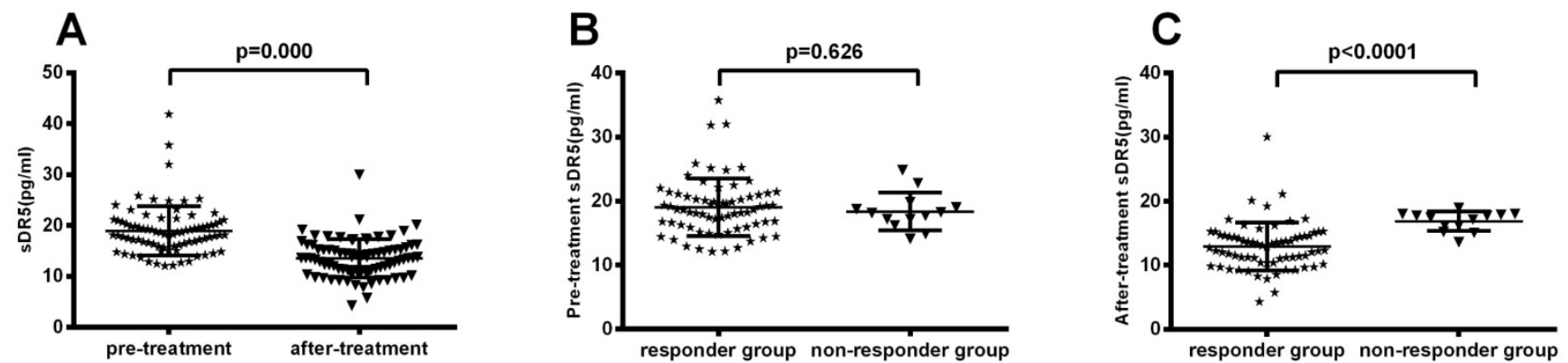

Figure 3. Association of pre- and post-treatment levels of serum sDR5 in SCLC patients. The blood samples were obtained from 82 SCLC patients before and after treatment and then subjected to analysis of sDR5 levels using the ELISA. (A) Association of post-treatment levels of serum sDR5 in SCLC patients, with responders vs. non-responders, $\mathrm{P}<0.0001$ using Student's $t$ test. (B) Association of pretreatment levels of serum sDR5 in SCLC patients with responders vs. non-responders, $\mathrm{P}=0.62$ using Student's $t$ test. 
Table 2. Univariate and multivariate analysis

\begin{tabular}{lllllll}
\hline Characteristics & \multicolumn{2}{l}{ Univariate } & \multicolumn{5}{l}{ Multivariate } \\
\hline & HR & $95 \% \mathrm{CI}$ & $p$-value & HR & $95 \% \mathrm{CI}$ & $p$-value \\
\hline $\begin{array}{l}\text { Gender } \\
\text { (male vs. female) }\end{array}$ & 1.27 & $0.77-1.10$ & 0.33 & & & \\
$\begin{array}{l}\text { Age (yrs.) } \\
(<65 \text { vs. } \geq 65)\end{array}$ & 1.63 & $0.99-1.69$ & 0.053 & & & \\
$\begin{array}{l}\text { PS } \\
(0-1 \text { vs. 2-3) }\end{array}$ & 6.17 & $3.32-11.45$ & $<0.001$ & 2.30 & $1.13-4.68$ & 0.021 \\
$\begin{array}{l}\text { Clinical Stage (VALSG) } \\
\text { (LS-SCLC vs. ED-SCLC) }\end{array}$ & 3.16 & $1.87-5.33$ & $<0.001$ & 0.48 & $0.54-1.63$ & 0.044 \\
$\begin{array}{l}\text { T Stage } \\
(1+2 \text { vs. 3) }\end{array}$ & 0.95 & $0.58-1.55$ & 0.84 & & & \\
$\begin{array}{l}\text { N Stage } \\
\text { (negative vs. positive) }\end{array}$ & 0.55 & $0.33-0.91$ & 0.022 & 0.85 & $0.49-1.48$ & 0.57 \\
$\begin{array}{c}\text { Pre-sDR5 } \\
\text { (negative vs. positive) }\end{array}$ & 0.75 & $0.46-1.22$ & 0.24 & & & \\
$\begin{array}{c}\text { After-sDR5 } \\
\text { (negative vs. positive) }\end{array}$ & 3.944 & $2.306-6.745$ & $<0.001$ & 2.30 & $1.04-5.07$ & 0.039 \\
\begin{tabular}{l} 
Smoking history \\
\hline
\end{tabular} & 0.33 & $0.19-0.56$ & $<0.001$ & 0.55 & $0.30-1.00$ & 0.051 \\
\hline
\end{tabular}

CI, confidence interval; HR, hazard ratio; PS, performance status; VALSG, Veterans Administration Lung Cancer Study Group staging system; sDR5, soluble death receptor 5 .

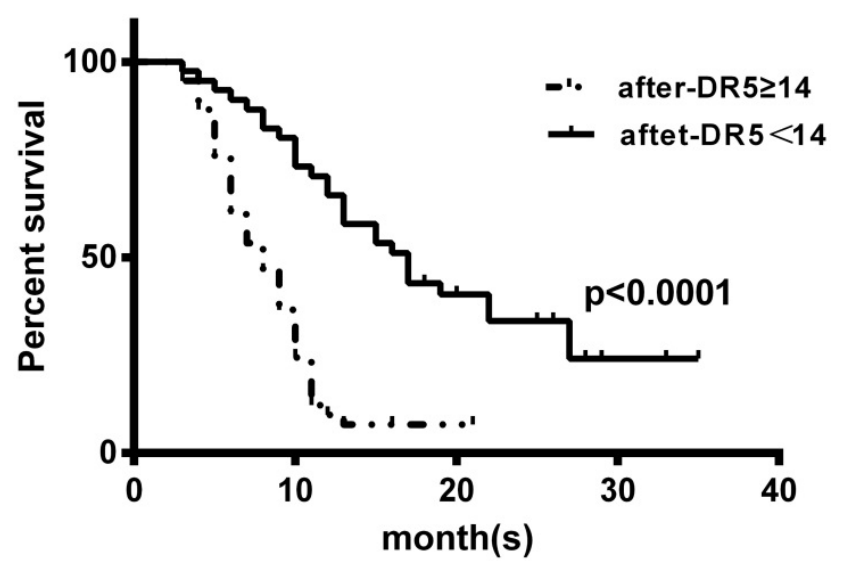

Figure 4. Kaplan-Meier curves stratified by the post-treatment levels of serum sDR5. The blood samples were obtained from SCLC patients after treatment and then subjected to ELISA analysis of sDR5 levels. To prepare the Kaplan-Meier curves, we selected the cut-off value of sDR5 point at $14 \mathrm{pg} / \mathrm{ml}$ according to the receiver operating characteristics analysis of $14 \mathrm{pg} / \mathrm{ml} . \mathrm{P}<0.0001$ analyzed by using the log-rank test.

\section{Discussion}

SCLC remains a deadly disease, even after decade-long advancements in early detection and improvement of treatment options [29, 30]. In the current study, we found that the serum soluble DR5 level was upregulated in SCLC patients compared with that of the healthy controls. Thus, future studies with prospective patients could verify our current data so that serum sDR5 level could be used as an effective biomarker to assess the treatment responses and prognosis of SCLC patients.

Apoptosis is a mechanism in multicellular organisms to maintain homeostasis; for example, it is believed that children between 8 and 14 of age lose approximately 20 to 30 billion cells a day due to apoptosis [31]. Apoptosis is a controlled process whereby cell shrinkage, nuclear fragmentation, chromatin condensation, and chromosomal DNA fragmentation are controlled by gene expression and activation through both intrinsic and extrinsic apoptotic pathways [12-16]. The TRAIL and TRAIL receptors can directly induce apoptosis of tumor cells through activation of the cell surface death receptors, while preserving normal cells [32, 33]. The TRAIL-DR5 apoptosis pathway was shown to play an important role in regulation of cell apoptosis [19] and associated with cancer development and progression $[15,16]$ as well as showed clinical significance of DR5 expression in human cancers. For instance, DR5 expression was independent prognostic indicator in bladder cancer and associated with better responses to treatment of patients [34].

Nuclear and cytoplasmic DR5 expression predicted survival of NSCLC after chemotherapy [15], whereas high DR5 expression was associated with worse disease-specific survival of renal cell carcinoma patients [35]. The pretreatment level of serum sDR5 was higher in NSCLC patients than that of healthy controls, but the post-treatment level of serum sDR5 failed to predict treatment responses or progression-free survival of these NSCLC patients [26]. In our current study, we confirmed high levels of serum sDR5 in SCLC patients versus healthy controls. Our data also demonstrated that serum sDR5 was able to predict treatment responses of SCLC patients. All of these studies, including our own, demonstrated opposite effects of DR5 expression on cancer progression and the treatment responses. Thus further studies are needed. Overexpression of DR5 in cancer tissues could be due to fast-growing tumor cells lacking an appropriate blood supply, resulting in tumor cell death through induction of either apoptosis or necrosis and leading to increase in upregulation of apoptosis-related proteins.

In our current study, we identified $14 \mathrm{pg} / \mathrm{ml}$ as the cut-off value for high versus low levels of serum sDR5. Depending on the sample size and sensitivity of each ELISA, this cut-off value could vary; therefore, a future study with a large sample size could confirm our current data. Furthermore, the cellular origin of the increased serum sDR5 level is unknown. A previous study speculated that there was an association between sDR5 secretion and proliferation of tumor cells [15]. In our current study, we found that pretreatment level of serum sDR5 was associated with smoking history, VALSG stage, tumor size, and SCLC lymph node metastasis, indicating that increase in serum sDR5 level could be from tumor cells. Furthermore, our current data demonstrated that serum level of sDR5 was able to predict OS of SCLC patients, although proper detection of sDR5 levels in SCLC has not yet been established. 
In conclusion, our current study revealed a predictive value of serum sDR5 level in SCLC patients. However, a multi-center study with a large sample size will be required to validate our current data before sDR 5 can be used clinically as a prognostic and predictive marker for SCLC.

\section{Acknowledgements}

This work was supported in part by grants from The Medical and Health Science and Technology Development Plan of Shandong Province (\#2017WS790, \#2015WS0213), the Natural Science Foundation of Shandong Province (\#ZR2016HQ50), and the National Natural Science Foundation of China (\#81301868).

\section{Competing Interests}

The authors have declared that no competing interest exists.

\section{References}

1. Torre LA, Bray F, Siegel RL et al. Global cancer statistics, 2012. CA: a cancer journal for clinicians. 2015; 65:87-108.

2. Collins LG, Haines C, Perkel R et al. Lung cancer: diagnosis and management. American family physician. 2007; 75:56-63.

3. Rosti G, Bevilacqua G, Bidoli P et al. Small cell lung cancer. Annals of oncology: official journal of the European Society for Medical Oncology. 2006; 17 Suppl 2:ii5-10.

4. Shepherd FA, Crowley J, Van Houtte P et al. The International Association for the Study of Lung Cancer lung cancer staging project: proposals regarding the clinical staging of small cell lung cancer in the forthcoming (seventh) edition of the tumor, node, metastasis classification for lung cancer. Journal of thoracic oncology : official publication of the International Association for the Study of Lung Cancer. 2007; 2:1067-1077.

5. Midthum D. Clinical presentation of lung cancer. Lung cancer: principles and practice. 1996: 421-435.

6. van Meerbeeck JP, Fennell DA, De Ruysscher DK. Small-cell lung cancer. Lancet (London, England). 2011; 378:1741-1755.

7. Zhang Y, He J. The development of targeted therapy in small cell lung cancer. Journal of thoracic disease. 2013; 5:538-548.

8. Planchard D, Le Pechoux C. Small cell lung cancer: new clinical recommendations and current status of biomarker assessment. European journal of cancer (Oxford, England : 1990). 2011; 47 Suppl 3:S272-283.

9. Pelayo Alvarez M, Gallego Rubio O, Bonfill Cosp X et al. Chemotherapy versus best supportive care for extensive small cell lung cancer. The Cochrane database of systematic reviews. 2009: Cd001990.

10. Han S, Gao F, Yang W et al. Identification of an SCLC susceptibility rs7963551 genetic polymorphism in a previously GWAS-identified 12p13.33 RAD52 lung cancer risk locus in the Chinese population. International journal of clinical and experimental medicine. 2015; 8:16528-16535.

11. Yang H, Song Y. Structural Insight for Roles of DR5 Death Domain Mutations on Oligomerization of DR5 Death Domain-FADD Complex in the Death-Inducing Signaling Complex Formation: A Computational Study. Journal of molecular modeling. 2016; 22:89.

12. Hanahan D, Weinberg RA. The hallmarks of cancer. Cell. 2000; 100:57-70.

13. Nagata S. Apoptosis by death factor. Cell. 1997; 88:355-365.

14. Amm HM, Oliver PG, Lee $\mathrm{CH}$ et al. Combined modality therapy with TRAIL or agonistic death receptor antibodies. Cancer biology \& therapy. 2011; 11:431-449.

15. Leithner K, Stacher E, Wurm R et al. Nuclear and cytoplasmic death receptor 5 as prognostic factors in patients with non-small cell lung cancer treated with chemotherapy. Lung cancer (Amsterdam, Netherlands). 2009; 65:98-104.

16. Spierings DC, de Vries EG, Timens W et al. Expression of TRAIL and TRAIL death receptors in stage III non-small cell lung cancer tumors. Clinical cancer research : an official journal of the American Association for Cancer Research. 2003; 9:3397-3405

17. Wiley SR, Schooley K, Smolak PJ et al. Identification and characterization of a new member of the TNF family that induces apoptosis. Immunity. 1995; 3:673-682

18. Huang Y, Sheikh MS. TRAIL death receptors and cancer therapeutics. Toxicology and applied pharmacology. 2007; 224:284-289.

19. Kelley RF, Totpal K, Lindstrom SH et al. Receptor-selective mutants of apoptosis-inducing ligand $2 /$ tumor necrosis factor-related apoptosis-inducing ligand reveal a greater contribution of death receptor (DR) 5 than DR4 to apoptosis signaling. The Journal of biological chemistry. 2005; 280:2205-2212.

20. Koornstra JJ, Jalving M, Rijcken FE et al. Expression of tumour necrosis factor-related apoptosis-inducing ligand death receptors in sporadic and hereditary colorectal tumours: potential targets for apoptosis induction. European journal of cancer (Oxford, England : 1990). 2005; 41:1195-1202.

21. van Geelen CM, Westra JL, de Vries EG et al. Prognostic significance of tumor necrosis factor-related apoptosis-inducing ligand and its receptors in adjuvantly treated stage III colon cancer patients. Journal of clinical oncology : official journal of the American Society of Clinical Oncology. 2006; 24:4998-5004.

22. Reesink-Peters N, Hougardy BM, van den Heuvel FA et al. Death receptors and ligands in cervical carcinogenesis: an immunohistochemical study. Gynecologic oncology. 2005; 96:705-713.

23. Cooper WA, Kohonen-Corish MR, Zhuang $\mathrm{L}$ et al. Role and prognostic significance of tumor necrosis factor-related apoptosis-inducing ligand death receptor DR5 in nonsmall-cell lung cancer and precursor lesions. Cancer. 2008; 113:135-142.

24. Elrod HA, Fan S, Muller S et al. Analysis of death receptor 5 and caspase-8 expression in primary and metastatic head and neck squamous cell carcinoma and their prognostic impact. PloS one. 2010; 5:e12178.

25. Lu M, Lawrence DA, Marsters S et al. Opposing unfolded-protein-response signals converge on death receptor 5 to control apoptosis. Science (New York, NY). 2014; 345:98-101.

26. $\mathrm{Yu} \mathrm{X}, \mathrm{Du}$ J, Zhai C et al. Clinical significance of serum soluble death receptor 5 concentration in locally advanced non-small cell lung cancer patients. Oncology letters. 2014; 8:1333-1339.

27. Micke P, Faldum A, Metz T et al. Staging small cell lung cancer: Veterans Administration Lung Study Group versus International Association for the Study of Lung Cancer--what limits limited disease? Lung cancer (Amsterdam, Netherlands). 2002; 37:271-276.

28. Therasse P, Arbuck SG, Eisenhauer EA et al. New guidelines to evaluate the response to treatment in solid tumors. European Organization for Research and Treatment of Cancer, National Cancer Institute of the United States, National Cancer Institute of Canada. Journal of the National Cancer Institute. 2000; 92:205-216.

29. Gong J, Salgia R. Managing Patients With Relapsed Small-Cell Lung Cancer. Journal of oncology practice. 2018; 14:359-366.

30. Zhao H, Ren D, Liu H et al. Comparison and discussion of the treatment guidelines for small cell lung cancer. Thorac Cancer. 2018; 9:769-774.

31. Karam JA: Apoptosis in Carcinogenesis and Chemotherapy. Netherlands. In.: Springer. ISBN; 2009.

32. Lemke J, von Karstedt S, Zinngrebe J et al. Getting TRAIL back on track for cancer therapy. Cell death and differentiation. 2014; 21:1350-1364.

33. Romeo C, Weber MC, Zarei M et al. HuR Contributes to TRAIL Resistance by Restricting Death Receptor 4 Expression in Pancreatic Cancer Cells. Molecular cancer research : MCR. 2016; 14:599-611.

34. Li Y, Jin X, Li J et al. Expression of TRAIL, DR4, and DR5 in bladder cancer: correlation with response to adjuvant therapy and implications of prognosis. Urology. 2012; 79:968.e967-915.

35. Macher-Goeppinger S, Aulmann S, Tagscherer KE et al. Prognostic value of tumor necrosis factor-related apoptosis-inducing ligand (TRAIL) and TRAIL receptors in renal cell cancer. Clinical cancer research : an official journal of the American Association for Cancer Research. 2009; 15:650-659. 